\title{
A Case of Podocytic Infolding Glomerulopathy with Focal Segmental Glomerulosclerosis
}

\author{
Akira Iguchi $^{\mathrm{a}}$ Ayako Sohma $^{\mathrm{a}}$ Hajime Yamazaki ${ }^{\mathrm{a}}$ Tomoyuki Ito $^{\mathrm{a}}$ \\ Takako Saeki $^{a}$ Yumi Ito $^{b}$ Naohumi Imai ${ }^{b}$ Yutaka Osawa $^{b}$ \\ Ichiei Narita ${ }^{b}$ \\ ${ }^{a}$ Department of Internal Medicine, Nagaoka Red Cross Hospital, Nagaoka, and ${ }^{b}$ Division \\ of Clinical Nephrology and Rheumatology, Niigata University Graduate School of Medical \\ and Dental Sciences, Niigata, Japan
}

\section{Key Words}

Podocytic infolding glomerulopathy · Focal segmental glomerulosclerosis · Microspheres ·

Podocyte

\begin{abstract}
Podocytic infolding glomerulopathy (PIG) has been proposed as a new disease entity. A 14year-old girl underwent renal biopsy at our institution because of a chance finding of proteinuria. Light microscopic findings revealed a minor glomerular abnormality, but under a higher magnification, after periodic acid methenamine silver staining, a bubbling appearance in the glomerular basement membrane (GBM) was observed. An electron microscopic examination revealed microspheres in the GBM, which were sparse but global. The patient was diagnosed as having PIG. After 3 years, her urinary protein had increased and a second biopsy was performed, showing focal segmental glomerulosclerosis in addition to a lesser degree of podocytic infolding than at the first biopsy. This is the first report of a case complicated by a different type of glomerulonephritis after being diagnosed as PIG. A few cases of PIG are complicated by focal segmental glomerulosclerosis, suggesting several mechanisms for the disorder.

(c) 2013 S. Karger AG, Basel
\end{abstract}

\section{Introduction}

Podocytic infolding glomerulopathy (PIG) has recently been proposed as a possible new pathological entity [1]. In PIG, microspheres or microtubular structures, or both, are 
associated with the infolding of cytoplasmic processes of podocytes into the glomerular basement membrane (GBM). It is not known whether this lesion indicates a new disease entity or a transient morphological finding of a well-known disease. However, several cases with an apparently unique microstructure showing podocytic infolding have been reported as PIG. Although most cases were diagnosed as lupus nephritis or another primary glomerulonephritis, based on light microscopic findings, 4 cases were diagnosed as a minor glomerular abnormality (MGA). We report a case of PIG with microspheres in the GBM that were diagnosed as MGA based on light microscopic findings. After 3 years, the patient had a nephrotic-range proteinuria and was diagnosed by a second renal biopsy as having focal segmental glomerulosclerosis (FSGS) with PIG. This is the first report of a case of PIG that was diagnosed as MGA based on the first light microscopic findings and, 3 years later, as complicated with FSGS.

\section{Case Report}

A 14-year-old girl was referred to our institution as an inpatient because of proteinuria. When she was 9 years old, she had urinary protein (UP) detected during a school physical examination and she was followed in the Pediatrics Department of our hospital. Her UP gradually increased to a level of $1.9 \mathrm{~g} /$ day, and at 14 years, she was referred to our division. Laboratory tests showed a UP level of $2.35 \mathrm{~g} /$ day, negative results for urinary sedimentation, and creatinine clearance of $111.9 \mathrm{ml} / \mathrm{min} / 1.73 \mathrm{~m}^{2}$. No cytopenia, hypocomplementemia, or elevated liver enzymes were found (table 1). Her physical findings were normal. Light microscopy of her first renal biopsy specimens showed a total of 14 glomeruli, 1 of which had global sclerosis; the others were MGAs. However, under light microscopy at a higher magnification under periodic acid methenamine silver (PAM) staining, the glomeruli had a bubbling appearance of diffuse glomerular loops (fig. 1). Immunofluorescent staining was negative for immunoglobulin, complement, and fibrinogen. Electron microscopy revealed microspheres in the GBM, which were sparse but global (fig. 2). The microstructures took various forms, and almost all of them were encircled with a unit membrane. There was no direct consecutive podocytic infolding in the GBM, nor any mesangial interposition. The endothelial cells were not swollen. Foot process effacement was scattered and there was no thickening of the GBM. This finding suggested the possibility of PIG. The patient was started on telmisartan $10 \mathrm{mg} /$ day. However, her UP level gradually increased to the nephrotic range by 16 years, and at 17 years of age, she underwent a second renal biopsy. Laboratory tests showed a UP level of $3.06 \mathrm{~g} /$ day, negative results for urinary sedimentation, and creatinine clearance of $156.5 \mathrm{ml} / \mathrm{min} / 1.73 \mathrm{~m}^{2}$. Antinuclear antibodies had changed to positive and the double-stranded deoxyribonucleic acid (dsDNA) level was elevated at $14 \mathrm{IU} / \mathrm{ml}$. Serum total protein level was $5.5 \mathrm{~g} / \mathrm{dl}$ and serum albumin level was $3.4 \mathrm{~g} / \mathrm{dl}$. No cytopenia, hypocomplementemia, or physical findings indicative of collagen disease were found (table 1). Light microscopy of the second renal biopsy specimens revealed 31 glomeruli, of which 4 had global sclerosis, 2 had segmental sclerosis with cellular variant, while the remainder were MGA. The bubbling appearance of glomerular loops was detected at higher magnification on PAM staining, as in the first biopsy, although their number had markedly decreased. Immunofluorescent staining was positive for IgM, C3, and C1q in the segmental sclerosis lesions, whereas all were negative in the other lesions. Electron microscopy revealed endothelial cell swelling and endocapillary cell proliferation in the segmental sclerotic lesions. Foot process effacement was global. The microspheres observed in the GBM were sparser than in the first biopsy (fig. 3). We diagnosed PIG complicated by FSGS. Prednisolone 
$40 \mathrm{mg} /$ day $(0.7 \mathrm{mg} / \mathrm{kg})$ was prescribed, and the UP level gradually decreased. After 9 months, the UP was less than $0.5 \mathrm{~g} /$ day.

\section{Discussion}

This case exhibited microspheres in the GBM on the electron microscopic findings at the first renal biopsy, although light microscopy showed only MGA. After 3 years, a second renal biopsy revealed FSGS cellular variant with microspheres. We diagnosed the patient as having PIG complicated by FSGS.

Joh et al. [1] reported 25 cases with microspheres and microtubular structures in the GBM as PIG. The light microscopic findings of PIG reveal a bubbling or stipple formation in association with an occasional spike formation on PAM staining. On electron microscopy, cytoplasmic processes of podocytes infold into the GBM, which involves thickening of the lamina densa. These microspheres or microtubular structures have a unit membrane and may be equivalent to a cytoplasmic membrane [1].

On the first biopsy, this case showed MGA in the light microscopic findings. In previous reports, 4 cases of PIG showed such findings. In 3 of them, of which 2 were diagnosed as rheumatoid arthritis and mixed connective tissue disease, and 1 exhibited lupus-like characteristics, immunofluorescent staining revealed immunoglobulin deposition [2]. The other case was diagnosed as minimal change nephrotic syndrome and PIG complicated by Sjögren's syndrome; immunoglobulin and complement were negative on immunofluorescent staining, and podocytic infolding and foot process effacement were found on electron microscopy [3]. Our case was different from the former 3 cases because immunoglobulin and complement were both negative, and from the latter case because of the clinical findings and scattered foot process effacement.

The second biopsy in our case showed PIG complicated by FSGS with fewer findings of podocytic infolding. Previous reports include 4 cases of PIG complicated by FSGS [4-7]. One of them, who had serial biopsies, was diagnosed after the first biopsy as having PIG and FSGS associated with vesico-ureteral reflux (VUR) [4]. After a urethral catheter had been in place for a year, a second biopsy was performed in order to evaluate the histological findings after treatment. The second biopsy revealed findings indicative of FSGS and the disappearance of almost all podocytic infolding.

There is the possibility that on the initial biopsy our patient had FSGS that could not be detected by renal biopsy because of the small number of lesions. The fact that the first biopsy revealed scattered foot process effacement and one global sclerosis has indicated the possibility of FSGS at the initial biopsy. Therefore, our case might have been diagnosed as PIG and FSGS at the first biopsy. The possibility of FSGS at the first biopsy cannot be denied. However, in the PIG case with FSGS due to VUR, the second biopsy showed less podocytic infolding than the first, as the UP decreased with improving VUR [4]. Therefore, in PIG complicated with FSGS, the amount of podocytic infolding would be in proportion to UP level. On the other hand, our case showed less podocytic infolding at the second biopsy even though the UP increased. This would be compatible with our case having had PIG without FSGS, rather than complicated with FSGS at the first biopsy. Moreover, it is more important to make clear whether podocyte infolding is a morphological phenomenon or a finding of a new disease entity. The previous report showed round microparticles in FSGS [8]. Especially in FSGS in which podocyte injury is evaluated as fundamental damage, podocyte infolding may be caused by a morphological phenomenon of FSGS. However, because of a decrease of these lesions despite an increased UP level at the second biopsy, the podocyte infolding may 


\begin{tabular}{l|l}
\hline DOI: 10.1159/000354591 & $\begin{array}{l}\text { ○ } 2013 \text { S. Karger AG, Basel } \\
\text { www.karger.com/cru }\end{array}$ \\
\hline
\end{tabular}

Iguchi et al:: A Case of Podocytic Infolding Glomerulopathy with Focal Segmental Glomerulosclerosis

be a new disease entity such as PIG which trends to associate with FSGS and collagen disease. If the podocytic infolding is a morphological phenomenon of FSGS, the podocytic infolding would be increased in proportion to increased UP. Some of the cases with podocyte infoldings, which until now have been believed to be a morphological phenomenon with other glomerulonephritis, would be a new disease entity. To discuss whether this lesion is PIG or a morphological phenomenon, it is necessary to accumulate and evaluate many similar cases.

The pathogenic mechanism of PIG is unknown. However, in collagen disease-associated PIG, the mechanism might involve antibody complement-mediated factors, which might be related to the role of special types of complement activation in situ on the microstructures, as developed on immune electron microscopic analysis $[9,10]$. However, some of these cases were negative for immunoglobulin and complement in the immunofluorescence findings. Similarly, collagen disease-irrelevant PIG also may have positive and negative findings. These findings also suggest that PIG is indeed a new disease entity. However, the pathogenic mechanism of PIG may not be single, but multiple. Further cases of PIG need to be analyzed in order to determine whether PIG is a new disease entity or a transient morphological finding of a well-known disease.

\section{Disclosure Statement}

The authors declare that no conflict of interest exists.

\section{References}

1 Joh K, Taguchi T, Shigematsu H, Kobayashi Y, Sato H, Nishi S, Katafuchi R, Nomura S, Fujigaki Y, Utsunomiya Y, Sugiyama H, Saito T, Makino H: Proposal of podocytic infolding glomerulopathy as a new disease entity: a review of 25 cases from nationwide research in Japan. Clin Exp Nephrol 2008;12:421-431.

2 Sato H, Saito T, Yoshinaga K: Intramembranous fine deposit disease associated with collagen disorder: a new morphological entity? Virchows Arch A Pathol Anat Histopathol 1992;420:447-451.

3 Sugiyama H, Maruyama M, Morinaga H, Inoue T, Takiue K, Kikumoto Y, Kinomura M, Sada KE, Akagi S, Kitamura S, Maeshima Y, Makino H: Unique microstructures and podocytic infolding in glomerular basement membrane associated with collagen diseases: a report of three cases. Clin Exp Nephrol 2008;12:450-454.

4 Matsuo T, Kobayashi Y, Nemoto N, Sano T, Kamata K, Shigematsu H: A nephritic case of vesicoureteral reflux representing focal segmental glomerulosclerosis associated with podocytic infolding lesions. Clin Exp Nephrol 2008;12:494-500.

5 Nazneen A, Nakashima Y, Zha Y, Le VT, Taguchi T, Nishioka K, Tadokoro M, Taura K: Unusual glomerulopathy with atypical thickening of glomerular basement membrane and intramembranous microparticles. Clin Exp Nephrol 2008;12:501-503.

6 Inoue Y, Kanda Y, Kinoshita C, Kanda C, Joh K: Intramembranous microspherical structures in focal segmental glomerulosclerosis: a case report. Clin Exp Nephrol 2008;12:504-508.

7 Yamada S, Masutani K, Katafuchi R, Fujigaki Y, Muranaka Y, Tsuruya K, Iida M: Focal segmental glomerulosclerosis with intramembranous vesicle-like microstructures and podocytic infolding lesion. Clin Exp Nephrol 2008;12:509-512.

8 Yoshikawa N, Cameron AH, White RH: Ultrastructure of the non-sclerotic glomeruli in childhood nephritic syndrome. J Pathol 1982;136:133-147.

9 Nakajima M, Hewitson TD, Mathews DC, Kincaid-Smith P: Localization of complement components in association with glomerular extracellular particles in various renal diseases. Virchows Arch A Anat Histopathol 1991;419:267-272.

10 Hinglais N, Kazatchkine MD, Bhakdi S, Appay MD, Mandet C, Grossetete J, Bariety J: Immunohistochemical study of the C5b-9 complex of complement in human kidneys. Kidney Int 1986;30:399-410. 


\begin{tabular}{l|l}
\hline DOI: 10.1159/000354591 & $\begin{array}{l}\text { ○ 2013 S. Karger AG, Basel } \\
\text { www.karger.com/cru }\end{array}$ \\
\hline
\end{tabular}

Iguchi et al.: A Case of Podocytic Infolding Glomerulopathy with Focal Segmental Glomerulosclerosis

Table 1. Laboratory data at the first and second renal biopsy

\begin{tabular}{lcc}
\hline & First renal biopsy & Second renal biopsy \\
\hline $\mathrm{Cr}, \mathrm{mg} / \mathrm{dl}$ & 0.45 & 0.55 \\
$\mathrm{BUN}, \mathrm{mg} / \mathrm{dl}$ & 10.0 & 11.8 \\
$\mathrm{Hb}, \mathrm{g} / \mathrm{dl}$ & 12.0 & 11.9 \\
$\mathrm{WBC}, / \mu \mathrm{l}$ & 5,100 & 8,200 \\
$\mathrm{Plt}, \times 10^{4} / \mu \mathrm{l}$ & 27.9 & 27.8 \\
$\mathrm{UP}, \mathrm{g} / \mathrm{day}$ & 2.35 & 3.06 \\
$\mathrm{URBC}, / \mathrm{hpf}$ & $<1$ & $1-4$ \\
$\mathrm{UWBC}, / \mathrm{hpf}$ & $<1$ & $<1$ \\
$\mathrm{ANA}$ & $<40$ & 40 \\
$\mathrm{dsDNA}, \mathrm{IU} / \mathrm{ml}$ & - & 14 \\
$\mathrm{C} 3, \mathrm{mg} / \mathrm{dl}$ & 111 & 107 \\
$\mathrm{C} 4, \mathrm{mg} / \mathrm{dl}$ & 25 & 24 \\
$\mathrm{CH} 50, \mathrm{U} / \mathrm{ml}$ & 50 & 47 \\
$\mathrm{IgG}, \mathrm{mg} / \mathrm{dl}$ & 931 & 727 \\
$\mathrm{IgA}, \mathrm{mg} / \mathrm{dl}$ & 201 & 163 \\
$\mathrm{IgM}, \mathrm{mg} / \mathrm{dl}$ & 89 & 86 \\
$\mathrm{CRP}, \mathrm{mg} / \mathrm{dl}$ & $<0.05$ & $<0.05$ \\
$\mathrm{Ccr}, \mathrm{ml} / \mathrm{min} / 1.73 \mathrm{~m}^{2}$ & 111.9 & 156.5 \\
\hline
\end{tabular}

$\mathrm{Cr}=$ Creatinine; $\mathrm{BUN}=$ blood urea nitrogen $\mathrm{Hb}=$ Hemoglobin; $\mathrm{WBC}=$ white blood cells; Plt = platelets; URBC = urinary red blood cells; $\mathrm{UWBC}=$ urinary white blood cells; $\mathrm{ANA}=$ antinuclear antibody; $\mathrm{CRP}=$ C-reactive protein; $\mathrm{Ccr}=$ creatinine clearance. 


\section{Case Reports in \\ Nephrology and \\ Urology}

\begin{tabular}{l|l}
\hline Case Rep Nephrol Urol 2013;3:110-116 \\
\hline DOI: 10.1159/000354591 & $\begin{array}{l}\text { @ 2013 S. Karger AG, Basel } \\
\text { www.karger.com/cru }\end{array}$ \\
\hline
\end{tabular}

Iguchi et al.: A Case of Podocytic Infolding Glomerulopathy with Focal Segmental Glomerulosclerosis
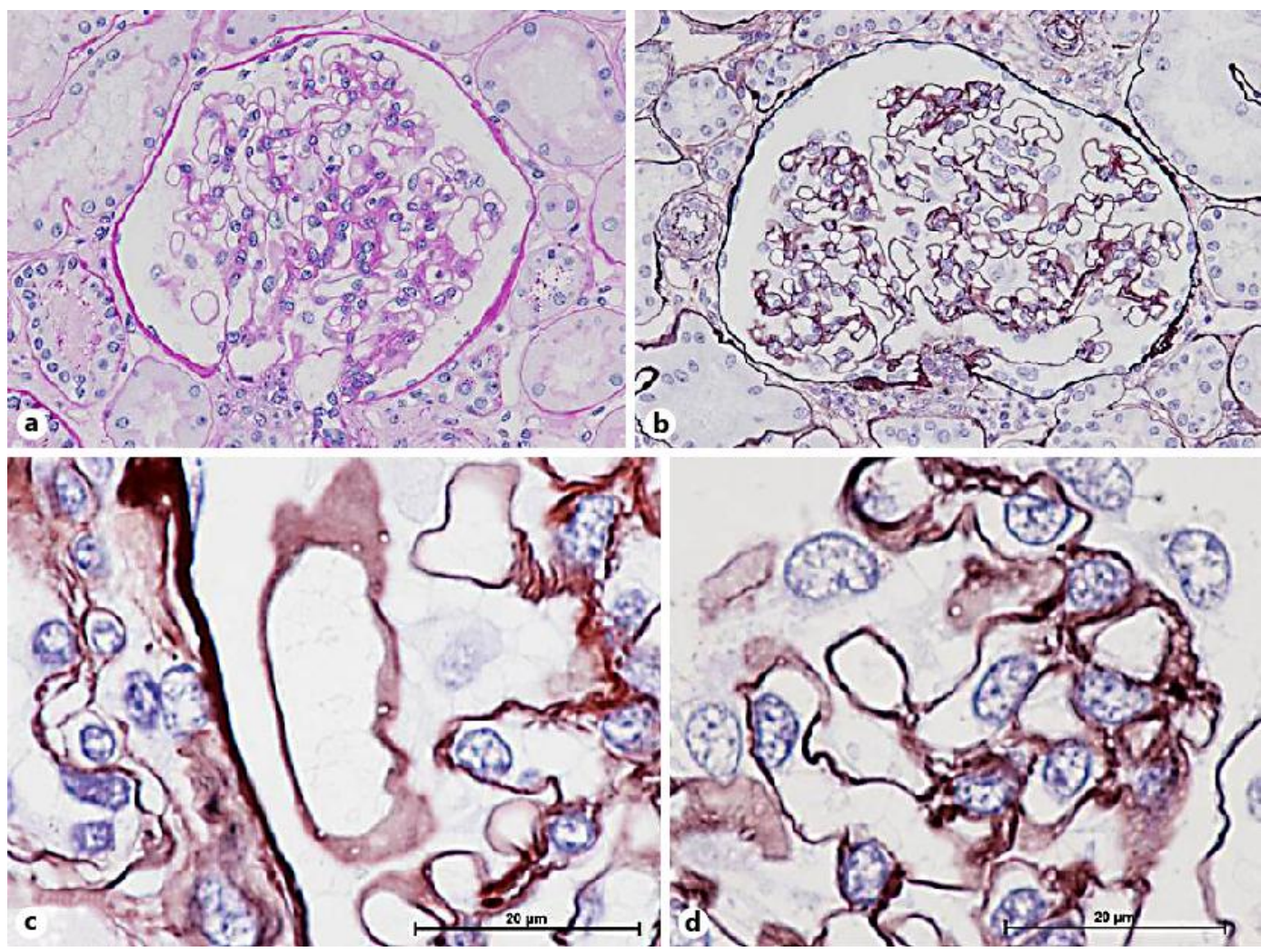

Fig. 1. Light microscopic findings at first renal biopsy. At low magnification, the glomerulus shows MGAs. At higher magnification, bubbling of the GBM is revealed by PAM stain. a Periodic acid Schiff (PAS) stain, $\times 400$. b PAM stain, $\times 400$. c, d PAM stain, $\times 2,000$.

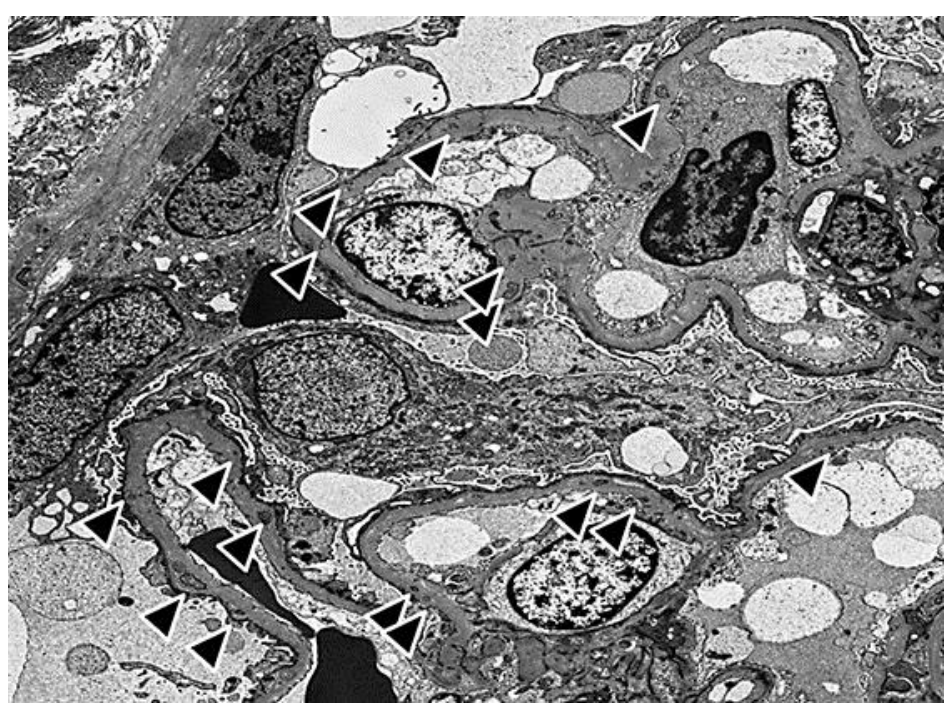

Fig. 2. Electron microscopic findings at the first renal biopsy. Several microspheres are shown in the GBM of a capillary loop (arrowheads). 
Iguchi et al:: A Case of Podocytic Infolding Glomerulopathy with Focal Segmental Glomerulosclerosis

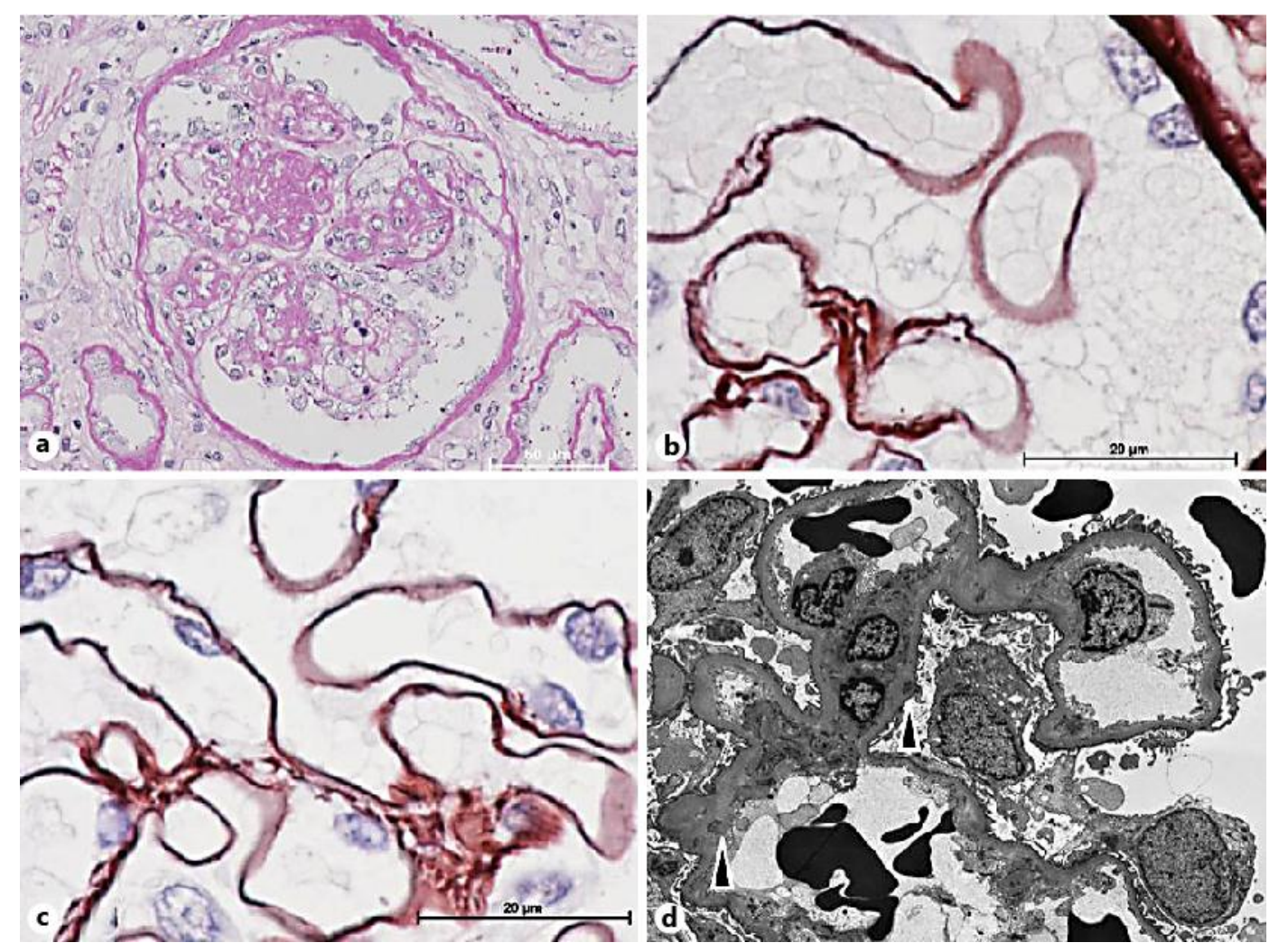

Fig. 3. Microscopic findings at the second renal biopsy. Light microscopic findings show segmental sclerosis with cellular variant. At higher magnification, bubbling of the GBM is revealed by PAM stain. At electron microscopy, microspheres are shown in the GBM (arrowheads), although microspheres are sparser than at the first biopsy. a Light microscopic findings, PAS stain, $\times 400$. b, c Light microscopic findings, PAM stain, $\times 2,000$. $d$ Electron microscopic findings. 